\title{
Coalescence and splitting of confined droplets at microfluidic junctions
}

\author{
G. F. Christopher, J. Bergstein, N. B. End, M. Poon, C. Nguyen and S. L. Anna* \\ Received 29th July 2008, Accepted 12th December 2008 \\ First published as an Advance Article on the web 23rd January 2009 \\ DOI: 10.1039/b813062k
}

The ability to merge two droplets is an important component of droplet-based lab-on-a-chip devices, yet flow-induced coalescence is difficult to achieve due to long film drainage times compared with relatively short residence times. We examine droplet collisions at a simple microfluidic T-junction and characterize the response for a wide range of droplet sizes and speeds. We find that three primary responses occur, where coalescence occurs easily at low collision speeds, smaller droplets traveling faster slip past one another without coalescing, and larger and faster droplets can break one another into multiple segments. The critical capillary number for coalescence agrees well with previously reported scaling for isolated droplet pairs when local curvature and speed are taken into account. The critical capillary number for splitting of droplets agrees well with a previously reported stability condition for individual droplets stretching in an extensional flow. Quantifying the necessary conditions for coalescence and non-coalescence behavior should enable the informed design of lab on chip devices based on discrete liquid segments.

\section{Introduction}

A promising recent development in lab-on-a-chip devices is the use of discrete liquid segments such as droplets and plugs to transport liquid samples within a device. Droplets offer numerous tangible advantages over single-phase flow. For example, the liquid interface separates the contents from contaminants and device constituents, the circulating flow in the droplet can lead to rapid mixing, and droplet motion can be used to monitor the kinetics of reactions occurring within. ${ }^{1}$ These advantages have been exploited in applications such as DNA analysis, ${ }^{2}$ protein crystallization, ${ }^{3}$ and other types of bio-analysis. ${ }^{4}$ In order to realize successful droplet-based devices, multiple "unit operations" must be combined and integrated, including generation of droplets, ${ }^{4,5}$ fission, ${ }^{6}$ sorting, ${ }^{7}$ and mixing within droplets. ${ }^{1,7}$ In addition, the ability to merge two droplets with different contents is critical to the success of these devices.

Flow-induced coalescence of droplets in microfluidic channels has been difficult to achieve due to relatively long film drainage times compared with short residence times. ${ }^{89}$ Additionally, surfactants are often used to stabilize droplets, hindering coalescence. ${ }^{6,10}$ In microfluidics, flow-induced coalescence in droplet streams has been demonstrated in long slowly diverging channels. ${ }^{11}$ As droplets travel along the diverging section, the spacing between them decreases, allowing some of the droplets to make contact and coalesce. A drawback to this method is a lack of control over which droplets collide. Flow-induced coalescence has also been achieved using a trifurcating junction to aid film drainage. ${ }^{12}$ In this case, initial droplet spacing and device geometry control which droplets merge.

Active control of droplet coalescence in microfluidics has been achieved using electric fields to bring together oppositely charged droplets in T-junctions ${ }^{8}$ and straight channels., ${ }^{9,13,14}$ Magnetic

Department of Mechanical Engineering, Carnegie Mellon University, Pittsburgh, PA, 15213,USA. E-mail: sanna@cmu.edu fields have also been used with success. ${ }^{15}$ Although these methods offer significant control compared with previous efforts to implement flow-induced coalescence, electromagnetic fields place strict requirements on the composition of the fluid within the droplet, and they can damage fragile contents such as DNA and proteins. The ability to passively control the merging of two droplets by exploiting fluid dynamics is desirable since it is simple to integrate into complex devices and places few restrictions on the fluid constituents.

Flow-induced coalescence is well studied outside the realm of microfluidics. For example, collisions of isolated droplet pairs in well-defined flow fields have been examined extensively. Experimentally, computerized four-roll mill devices allow control and visualization of droplet collisions in a viscous reservoir. ${ }^{16}$ When two spherical droplets make contact, they initially slide past one another, rotating such that the line connecting the droplet centers is no longer parallel to the flow axis. Over time, there is drainage of the thin liquid film separating the droplets. The excess capillary pressure in the film drives drainage, and the rate of drainage is moderated by the viscous stresses within the film, which are determined by factors such as the speed of the collision and the viscosity of the liquid in the film. ${ }^{17}$ Thus, in the absence of inertia, as is typical for microfluidic devices, ${ }^{18}$ a controlling parameter for coalescence is the capillary number $\mathrm{Ca}$ describing the relative magnitude of viscous stresses and capillary pressure. Once the film becomes thin enough, van der Waals and other intermolecular forces govern the film thinning. If the droplets remain in contact long enough, the film ruptures and the droplets merge. Observations show that coalescence occurs when collisions are slow, corresponding to capillary numbers below a critical value of order $C a_{\mathrm{c}} \approx 10^{-2}$. Numerous experimental and numerical studies have characterized the film drainage time, the critical capillary number, and the angle at which droplets coalesce as a function of droplet size, ${ }^{19-21}$ viscosity ratio, ${ }^{19,20,22}$ overall droplet deformation, ${ }^{23,24}$ and the offset of the collision. ${ }^{25-27}$ In particular, $\mathrm{Hu}$ and co-workers ${ }^{19}$ show that the critical capillary 
number for coalescence in a predominantly extensional flow when collisions are nearly head-on exhibits power law dependence on droplet radius $a$ and viscosity ratio $\lambda \equiv \mu_{\mathrm{d}} / \mu_{\mathrm{c}}$, such that

$$
C a_{\mathrm{c}} \propto(\lambda)^{-0.41 \pm 0.06}(2 a)^{-0.82 \pm 0.03}
$$

where $\mu_{\mathrm{d}}$ and $\mu_{\mathrm{c}}$ represent the droplet phase and matrix phase liquids, respectively. Additionally, the angle at which coalescence occurs varies with capillary number and viscosity ratio. For slow, head-on collisions at low viscosity ratio, coalescence occurs at $0^{\circ}$, before droplets have begun to rotate about one another. The coalescence angle increases with increasing viscosity ratio. Although a simple force balance suggests that coalescence should occur only for angles less than $45^{\circ}$, coalescence angles greater than $45^{\circ}$ are observed for larger viscosity ratios. Borrell et al. attribute this to a suction force that arises from separation of the thin film and leads to faster drainage. ${ }^{25}$

Relatively few studies have focused on coalescence behavior in tubes and channels, in which droplets can deform significantly on account of the confining boundaries, and the flow of the surrounding viscous liquid controls the speed and trajectory of each droplet. In a recent numerical study, Yan et al. show that two droplets passing side-by-side through a constriction in a capillary tube can deform significantly and slide past one another without coalescing. ${ }^{28}$ Similar to isolated droplet pairs, the co-flowing droplets coalesce only if the capillary number is low enough. In this case, the capillary number is defined as $\mathrm{Ca} \equiv$ $\mu_{\mathrm{c}} U / \sigma$, where $U$ is the velocity of the droplets, and $\sigma$ is the interfacial tension. In this case, the critical capillary number for coalescence agrees well with values for isolated droplets, both in magnitude and in scaling with parameters such as the viscosity ratio. $^{19}$

Numerous studies have focused on other aspects of the coalescence process. The literature is extensive; we give just a few examples here. For example, coalescence has been examined in simple shear flow, ${ }^{17,26,29-31}$ in bulk emulsions ${ }^{32-34}$ and in specific configurations such as coalescence through planar surfaces. ${ }^{35,36}$ Several studies have examined the dynamics of film drainage in detail, ${ }^{20,37-39}$ including via electrical conductance ${ }^{40}$ and ellipsometry $^{41}$ measurements. In general, the rate of film drainage increases with increasing capillary number. For example, Klaseboer et al. $^{39}$ estimate the drainage timescale for droplets approaching one another head-on at constant velocity $U$, where

$$
t_{\text {drain }} \approx 40 a \sqrt{\mu_{\mathrm{c}} / \sigma U}
$$

This estimate of the coalescence timescale agrees well with measurements for surfactant free droplets. Detailed measurements of film drainage can inform global measurements of coalescence criteria, since the rate of film drainage competes with the contact time of the droplets to determine whether droplets coalesce.

These results taken together suggest that coalescence can be robust in microfluidic devices, particularly since coalescence in a constricting tube is not dramatically different from that of isolated droplet pairs, even when the flow fields appear to be very different. We expect that coalescence will occur easily and reliably in microfluidic devices as long as collisions are slow enough, and that droplets will not coalesce at higher speeds. Since the magnitude and scaling of the critical capillary number with viscosity ratio is similar for isolated droplets and droplets in a constricting tube, we expect to maintain the same scaling in microchannels. However, confinement of the droplets within the microchannels should play an important role since droplets in lab-on-a-chip applications are typically plug-like. Thus, changing the droplet volume leads to length variations, which influence the contact time of the droplets but do not significantly alter the shape of the droplet near coalescence.

To test these hypotheses, we devise a simple method for obtaining flow-induced droplet coalescence in microchannels using no external fields. A T-shaped junction is used to direct two streams of droplets toward each other at a nominally constant approach velocity. By varying the size of the colliding droplets and the velocity of approach, we organize the coalescence and non-coalescence behavior in terms of the global dimensionless parameters, the capillary number and the droplet aspect ratio. We quantify the critical capillary number for coalescence as a function of the droplet size and the viscosity ratio. Additionally, we describe non-coalescence behaviors that are observed for higher speed conditions, and quantify the boundaries between the various coalescence and non-coalescence regimes.

\section{Materials and methods}

The microchannels used in the present study are molded in poly(dimethyl siloxane) elastomer (PDMS; Dow Sylgard 184) using standard soft lithography fabrication methods. ${ }^{42,43}$ To close the microchannels, we first coat a thin layer of PDMS onto a glass microscope slide, and then expose the surfaces of both the molded microchannel and the thin PDMS layer to ionizing air plasma (Harrick Scientific). Plasma treatment ensures that the two surfaces bond covalently when they come into contact. The thin layer of PDMS ensures uniform wetting on all microchannel surfaces, and the glass microscope slide provides stability. Prior to use, sealed microchannels are placed in a $180{ }^{\circ} \mathrm{C}$ oven for two hours to ensure that the microchannel surfaces have reverted to hydrophobic wetting.

A schematic diagram of the microchannel geometry used in this study is shown in Fig. 1. Two upstream T-shaped junctions generate droplets of water in the cross-flowing stream of immiscible oil as shown in inset A. Cross-flowing streams is a common and well-characterized method to generate droplets, in which the droplet sizes are controlled by adjusting the volumetric flow rate of the continuous phase liquid, the ratio of the volumetric flow rates of the two immiscible liquids, and the ratio of the inlet channel widths $w_{\mathrm{c}}$ and $w_{\mathrm{d}} \cdot{ }^{44-46}$ Using identical flow rates for each of the upstream $\mathrm{T}$-junctions, droplets with nominally equal volume and speed are generated. Downstream, the two uniform droplet streams collide head-on at a second T-junction and flow together into a common outlet channel with width equal to the inlet channel width $w_{\mathrm{o}}=w_{\mathrm{c}}$ as shown in inset B. The geometric parameters considered in this study are listed in Table 1. In particular, we select a fixed width of the main channel $w_{\mathrm{c}}=w_{\mathrm{o}}=100 \mu \mathrm{m}$ and vary the width of the dispersed phase inlet channel from $w_{\mathrm{d}}=50$ to $200 \mu \mathrm{m}$. The uniform depth of the rectangular microchannels considered in this study is $h=80 \mu \mathrm{m}$.

The dispersed phase liquids used are solutions of deionized water and glycerol, ranging from pure water to $99 \mathrm{wt} . \%$ glycerol. Viscosity values are measured using a Bohlin rheometer with 


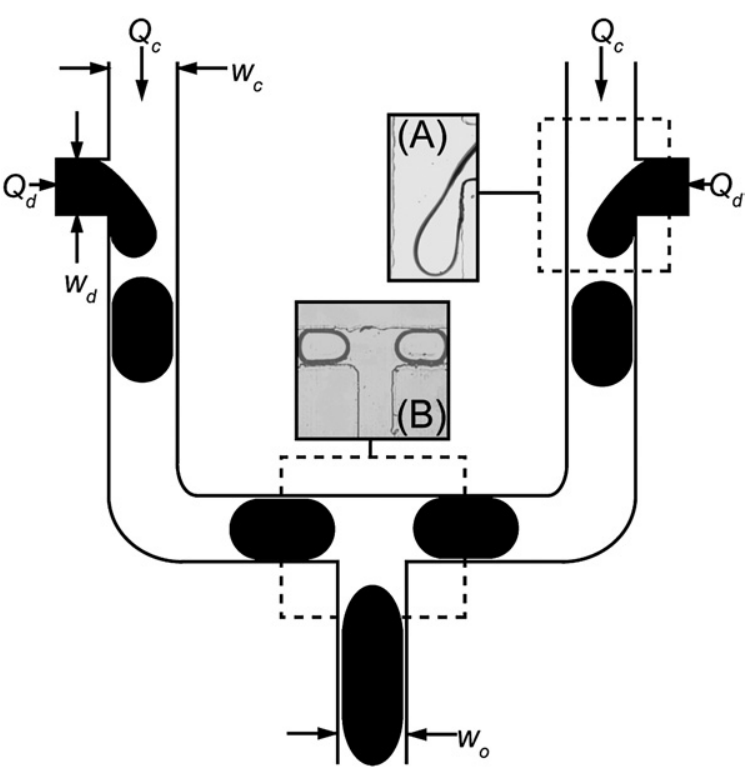

Fig. 1 Schematic diagram of microfluidic geometry used to characterize the response of droplets to collisions. Two upstream T-junctions are used to generate droplets as shown in inset (A). The resulting droplet streams interact at a third T-junction downstream and then flow together into a single outlet stream as shown in inset (B).

a double gap Couette cell geometry and are listed in Table 1. Low molecular weight silicone oil (Gelest) with a manufacturerreported viscosity of $100 \mathrm{mPa} s$ is used as the continuous phase liquid, leading to viscosity ratios ranging from $\lambda=\mu_{\mathrm{d}} / \mu_{\mathrm{c}}=0.01$ to 9.8. We use a Du Noüy ring tensiometer to measure the interfacial tension of the water-oil pairs, obtaining an average value of $\sigma=45.57 \pm 0.96 \mathrm{mN} / \mathrm{m}$ for pure water and silicone oil, and $\sigma=24.4 \pm 2.5 \mathrm{mN} / \mathrm{m}$ for all glycerol solutions paired with silicone oil. We assume that all the liquids are free of surfaceactive contaminants, and no additional surfactant is added.

The oil and water are driven into the device using independent syringe pumps (Harvard Apparatus PHD 2000). Prior to beginning an experiment, the device is primed by filling all microchannel segments with continuous phase oil. Once primed, the pump for the dispersed phase liquid is started. The pump for the continuous phase liquid is started after the dispersed phase liquid reaches the $\mathrm{T}$-junction. For the present experiments, volumetric flow rates range from $Q=0.5$ to $500 \mu \mathrm{l} / \mathrm{h}$. High-speed video of the colliding droplets is captured at the downstream $\mathrm{T}$ junction using a high-speed CMOS camera (IDT XS-4) mounted to an inverted light microscope (Nikon TE 2000U). Video is analyzed in post-processing to quantify the droplet size and the velocity of approach and to categorize the interaction between the two droplets. In addition, the distance between the droplet centroids and the angular relationship between the centroids is measured as a function of time during the approach.

\section{Results and discussion}

\section{Observations of droplet collisions at $\mathbf{T}$-junctions}

We systematically investigate the coalescence behavior of droplets confined within microchannels as a function of initial droplet size and speed, using the geometry shown in Fig. 1. We observe collision events at the downstream $\mathrm{T}$-junction and classify the observed behaviors. For the reference system in which the viscosity ratio is $\lambda=0.01$, we observe four primary responses of a droplet pair approaching the downstream T-junction: (1) no collision, (2) coalescence, (3) slipping, and (4) splitting. No collision occurs when there is a timing difference between the arrivals of the two droplets at the junction. A timing difference can occur when droplet production in coupled droplet generators is not synchronous, as Barbier et al. have characterized experimentally for a wide range of conditions ${ }^{47}$ Although we do not take steps to avoid timing differences, such non-synchronous behavior can be a limitation to the practical use of devices such as these. Cristobal et al. recently demonstrated a simple microfluidic bypass filter that effectively regulates droplet motion at junctions and could be used to control timing differences. ${ }^{48}$ When there is no timing difference and the droplets come into contact upon arrival at the junction, the two droplets can coalesce, merging to form a single droplet, they can slip past one another and travel downstream in a pair oriented end-to-end, or one droplet can force the other to split into two parts, where the three droplets proceed downstream in a triplet. Sample images depicting the three primary responses of colliding droplets when there is no timing difference are shown in Fig. 2(A)-(C). We label the droplet that arrives at the junction first the "receiving droplet" and the one that arrives second the "hitting droplet".

Along with the three primary responses of a colliding droplet pair, there are also several secondary behaviors that occur in a less regular manner including late coalescence, multiple splitting, and late splitting. In the case of late coalescence, depicted in Fig. 2(D), the droplets initially slip past one another as they pass through the T-junction and then coalesce just before the droplets have entered the downstream channel. In multiple splitting, depicted in Fig. 2(E), the droplets are typically relatively long plugs with aspect ratio greater than $\varepsilon>1.15$. In this case, the receiving droplet is initially split into two segments. As the segments slip past one another, the trailing segment of the original receiving droplet splits the trailing end of the hitting droplet. Late splitting, depicted in Fig. 2(F), is similar to late

Table 1 Physical properties of liquids used in experiments, along with dimensions of the microchannels used

\begin{tabular}{llll}
\hline Fluid System & Viscosity $[\mathrm{mPa} \mathrm{s}]$ & Device Dimensions [ $\mathrm{mm}]$ \\
\hline Dispersed Phase & Water-Glycerol Solutions & $1,9.6,36.7,62.7,130,490,980$ & $w_{\mathrm{c}} 100$ \\
Continuous Phase & Silicone Oil & 100 & $w_{\mathrm{d}} 50-200$ \\
Interfacial Tension $[\mathrm{mN} / \mathrm{m}]$ & & $45.57 \pm 0.96$ pure water & $w_{\mathrm{o}} 100$ \\
Volumetric Flow Rates $[\mu \mathrm{l} / \mathrm{h}]$ & & $24.4 \pm 2.5$ water-glycerol & $h 80$ \\
\hline
\end{tabular}




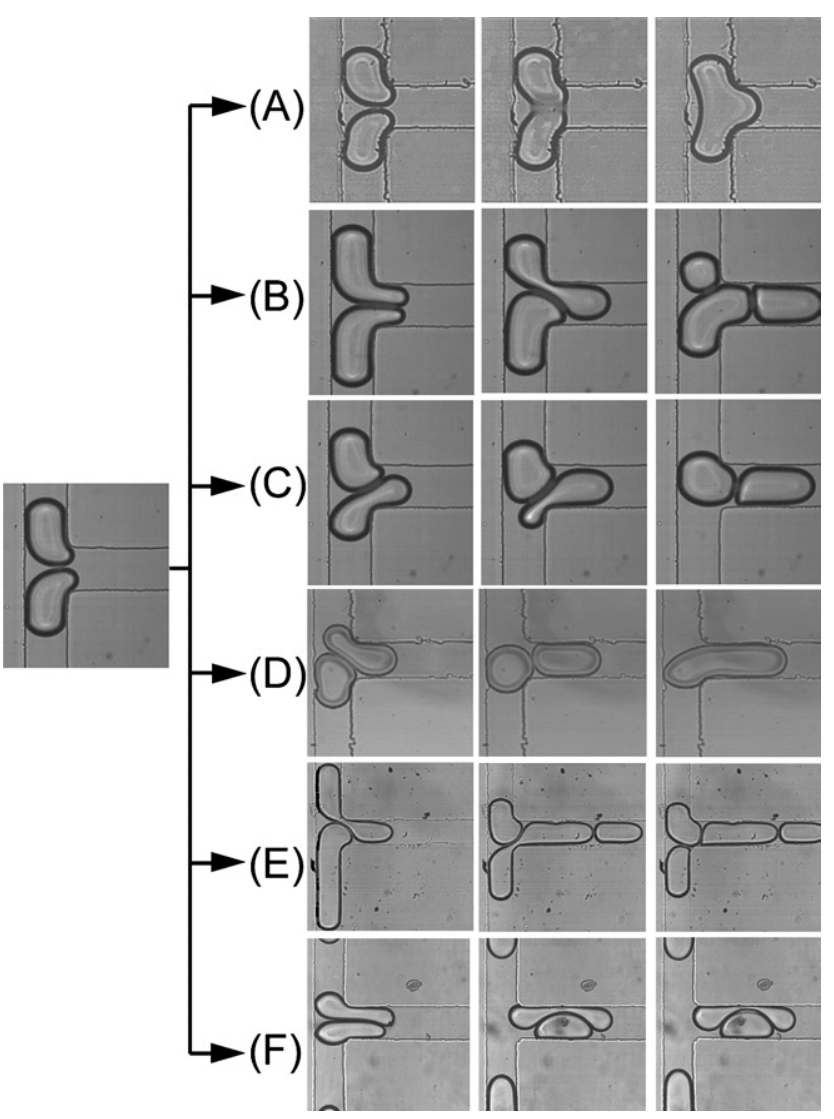

Fig. 2 Representative images of observed responses to droplet collisions at microfluidic junctions. Primary responses: (A) coalescence, (B) splitting, and (C) slipping. Secondary responses: (D) late coalescence, (E) multiple splitting, and (F) late splitting.

coalescence, occurring after the droplets have slipped past one another and entered the downstream channel.

\section{Droplet trajectories passing through the T-junction}

The trajectories of the approaching droplets exhibit a unique signature depending on the response to the collision. Fig. 3(A) shows a typical trace of the dimensionless distance between the centroids of the approaching droplets as a function of time for each of the three primary collision responses. The centroid-tocentroid distance is normalized by the width of the continuous phase channel $w_{c}$. The time axis is normalized by the characteristic residence time within the junction, $t_{\mathrm{c}} \equiv w_{\mathrm{c}} / U$, where $U$ is the approach velocity of the droplets. A constant approach velocity is expected based on constant inlet volumetric flow rates. The magnitude of the velocity depends on the total flow rate and the droplet size relative to the channel width in a complicated way. ${ }^{49}$ Rather than correlating the controlling parameters with the droplet speed, we simply measure the speed via image analysis for each collision. The angle between the droplet centroids is shown in Fig. 3(B) as a function of dimensionless time. For head-on collisions such as those considered in this study, the approach angle is defined as $\pi / 2$ radians. In the exit to the T-junction, the angle is zero radians if the centroid of the hitting droplet is upstream of the receiving droplet, and $\pi$ radians if it is downstream.

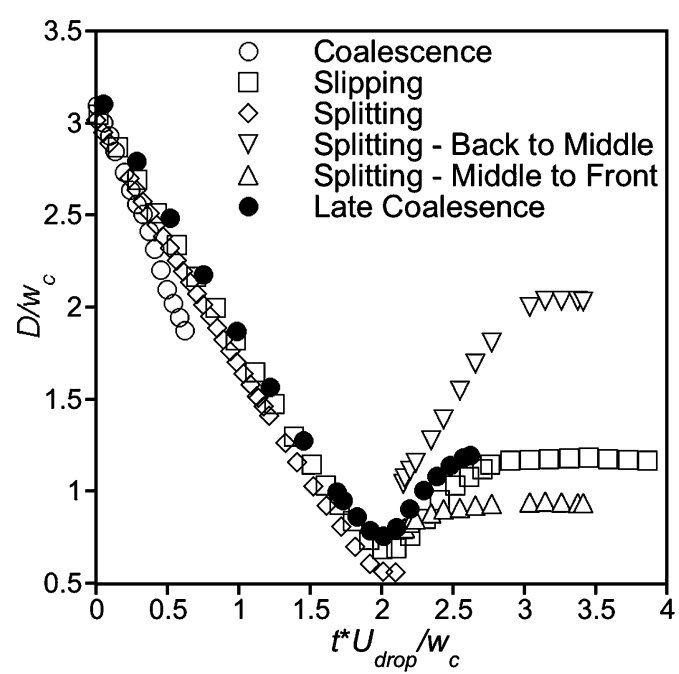

(A)

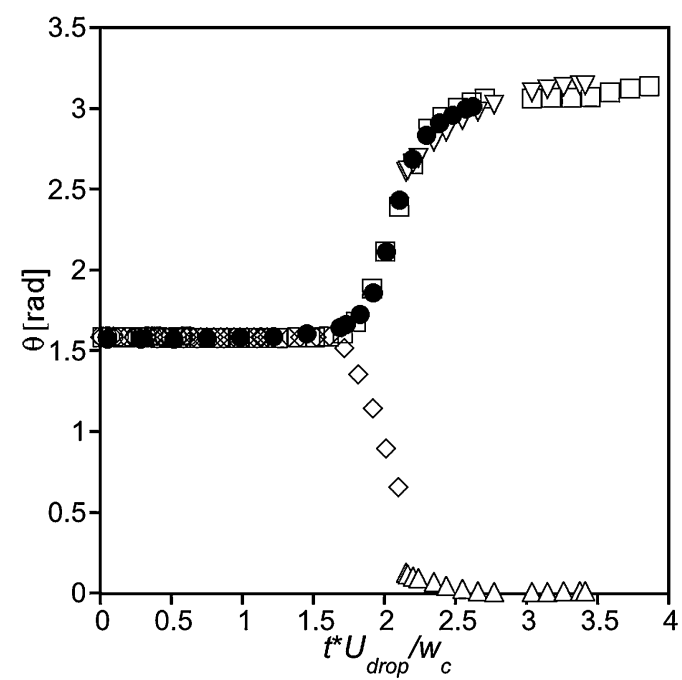

(B)

Fig. 3 Representative trajectories for droplet pairs approaching a junction. The plots show (A) the distance and (B) the angle between the centers of mass of the approaching droplets as a function of time for $(O)$ coalescence, $(\square)$ slipping, $(\diamond)$ splitting, and $(\bullet)$ late coalescence. In (A) the distance is normalized by the channel width, and in both plots the time axis is normalized by the channel width divided by the relative velocity of approach. In the case of splitting droplets, the segments are denoted $(\triangle)$ for the front droplet and $(\nabla)$ for the rear droplet. The same legend applies to both plots.

In all cases, the initial droplet trajectory is linear with time, consistent with the expected constant approach velocity. Furthermore, the initial trajectories are all similar when normalized as described above, indicating that the characteristic length and timescales are correct. At later times, each collision behavior occurs at a different dimensionless time, since the processes governing these timescales are very different from one another.

Specifically, droplet coalescence occurs relatively rapidly compared to other responses. In this case the distance between the centroids remains linear with time and the angle remains 
constant at $\pi / 2$ radians until the instant that the droplets merge. Thus, droplets undergo very little deformation during the coalescence process. By monitoring the time of contact of the droplets as they approach one another prior to coalescence, we observe an average coalescence time of $t_{\text {coal }}=8.87 \pm 0.97 \mathrm{~ms}$. The relatively large uncertainty on this measurement arises from an inability to resolve film thicknesses at these length and timescales well enough to accurately monitor the film drainage process. To capture these very rapid drainage times would require frame rates in excess of $12,000 \mathrm{fps}$, which is not feasible with our current experimental setup in combination with the needed spatial resolution. Nevertheless, our observed timescales are of the same order as the values obtained from the numerical simulations of Klaseboer et al., who analyzed the film drainage time for head-on collisions of droplets at constant approach velocity ${ }^{39}$ and obtained the estimate given in equation (2).

In contrast to coalescence, droplets slipping past one another or splitting into multiple parts undergo significant deformation as they squeeze through the T-junction after the initial collision, as shown in Fig. 2. In both cases the centroid distance reaches a minimum once the droplets come into contact, when the centroid angle is $3 \pi / 4$ radians. When the droplets slip past one another without breaking, the angle increases from $\pi / 2$ to $\pi$ radians and the centroid distance approaches a constant value as the droplets rearrange in the outlet channel. A constant centroid distance is maintained as the droplets travel downstream of the junction. This steady-state centroid distance is equal to the sum of the half-lengths of the droplets, since the two droplets remain in close contact as they travel downstream. When one droplet splits the other into two segments, the three resulting droplets travel downstream with constant centroid distances, aligned at 0 and $\pi$ radians from the central splitting droplet. Similar to slipping droplets, these distances are equal to the sum of the halflengths of the droplets in each pair.

Fig. 3 also depicts the trajectory of a droplet undergoing late coalescence, in which droplets first slip past one another and then coalesce downstream of the junction. In this case we note that the trajectories for a late coalescing droplet pair and a slipping droplet pair are nearly identical until the time that coalescence occurs.

We note that other coalescence studies also track the trajectories of colliding droplets, see for example Allan \& Mason $^{29}$ and Guido \& Simeone. ${ }^{31}$ We note for example that in the observations of Guido \& Simeone, the horizontal and vertical separation of two colliding droplets reflect the ability for the droplets to separate from one another spatially. By contrast, droplet trajectories in the microfluidic T-channel described are highly constrained, and droplets are forced to deform rather than move away from one another.

\section{Operating diagram for droplet collisions at a $\mathbf{T}$-junction}

The response of a droplet pair to a collision depends on the initial droplet size and the relative speed $U$ of the approaching droplets, where $U=U_{\text {hitting }}-U_{\text {receiving. We organize the collision }}$ responses on an operating diagram shown in Fig. 4 depicting the dimensionless droplet speed on the vertical axis and the dimensionless droplet size on the horizontal axis. The droplet speed is normalized by the capillary velocity $\sigma / \mu_{c}$ to form a capillary number $C a=\mu_{\mathrm{c}} U / \sigma$. The droplet size is characterized in terms of

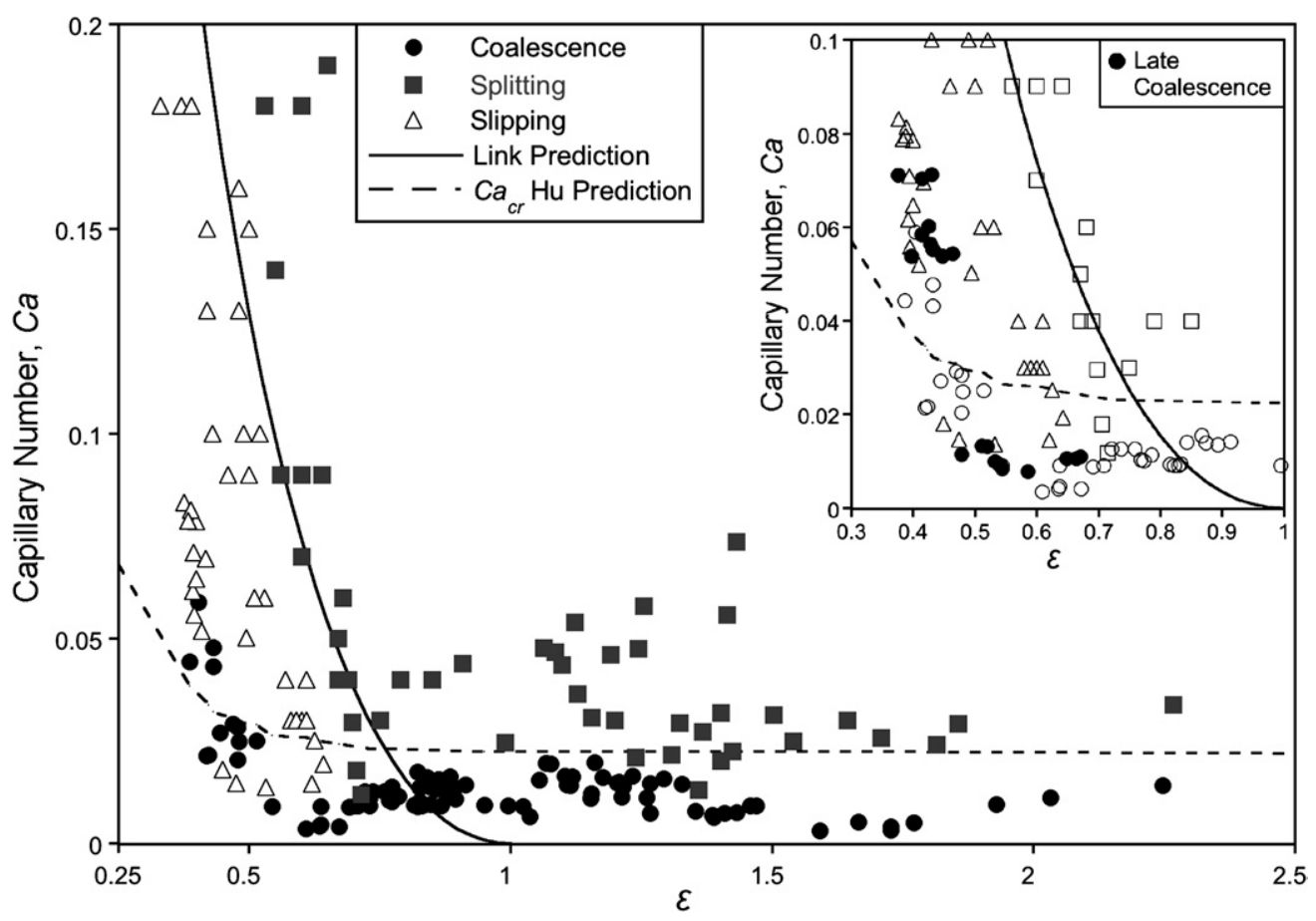

Fig. 4 Operating diagram organizing droplet behavior as a function of capillary number $\mathrm{Ca}$ and aspect ratio $\varepsilon$. Symbols denote the primary responses observed: $(\bullet)$ coalescence, $(\boldsymbol{\square})$ splitting, and $(\triangle)$ slipping. The dotted line represents the predicted critical capillary number for isolated droplets in a four-roll mill. ${ }^{19}$ The solid line represents the stability criterion for a droplet colliding at a junction given by Link et al. ${ }^{6}$ The inset shows a close-up view of the phase diagram at small aspect ratios to highlight the late coalescence behavior $(\boldsymbol{)})$ that occurs near the critical conditions for coalescence. 
the droplet width $w$ and length $l$, since the droplets formed in this study are predominantly plug-like due to their confinement within the rectangular channels. In this case the droplet length increases linearly with volume. ${ }^{44,50}$ Thus, the dimensionless droplet size is given by the aspect ratio $\varepsilon \equiv l / \pi w$, where $\pi w$ approximates the circumference of the droplet. The aspect ratio used in Fig. 4 is that of the receiving droplet.

Organizing a large number of collision responses in terms of the capillary number and aspect ratio over a range of flow conditions, we find that the three primary responses fall into distinct regions of the operating diagram. As Fig. 4 shows, droplets coalesce at low capillary number over the entire range of droplet sizes considered. Over most of this range there appears to be a critical capillary number in the neighborhood of $C a_{\mathrm{c}} \approx 0.02$. For capillary numbers larger than the critical value, small droplets slip past one another without coalescing, $c f$. Fig. 2(C). Larger droplets are split into two segments by the hitting droplet, cf. Fig. 2(B). The boundary between the slipping and splitting regimes shifts toward smaller aspect ratio as the capillary number increases.

\section{Critical capillary number for coalescence}

We record numerous collision events in order to quantify the critical capillary number for coalescence as a function of the droplet size and the viscosity ratio. The data shown in Fig. 4 suggests that $C a_{\mathrm{c}} \approx 0.02$ for aspect ratio larger than $\varepsilon>0.5$, where the viscosity ratio is $\lambda=0.01$. For comparison, consider values reported for isolated droplet pairs in an extensional flow, and droplet pairs colliding side-by-side in a constricting capillary tube. In the first case, we note that since the droplets we consider are 'plug-like' and fill the microchannel cross-section, the channel half-width $w_{\mathrm{c}} / 2$ approximates the curvature of the droplets at the point of collision. Thus, we compare our observations with collisions of isolated droplet pairs with diameter similar to the fixed channel width $w_{\mathrm{c}}=100 \mu \mathrm{m}$. Hu et al. report a critical value of $C a_{\mathrm{c}}=0.022$ for $100 \mu \mathrm{m}$ droplets colliding in a predominantly extensional flow at a viscosity ratio of $\lambda=$ $0.01 .{ }^{19}$ The estimate of $\mathrm{Hu}$ et al. is shown on Fig. 4 as a dashed line, for aspect ratio $\varepsilon>0.5$. Similarly, Yan et al. report values in the range of $C a_{\mathrm{c}} \approx 0.05$ to 0.064 for droplet pairs squeezing sideby-side through a constriction in a tube. ${ }^{28}$ Thus, the critical capillary number for coalescence of plug-like droplets at a microfluidic $\mathrm{T}$-junction is consistent with values reported for other flow configurations in which coalescence occurs. We note that these comparisons correspond to flow fields that are significantly different from one another, where isolated droplets collide in a relatively simple linear extensional flow, while the simulations of Yan et al. examine droplets contacting one another in a more complicated side-by-side configuration. The microfluidic T-channel configuration considered here contains elements of these flow fields, in which the droplets collide headon as they do for the extensional flow, albeit at constant speed, but then the droplets re-orient and slip past one another similar to the configuration of Yan et al. The fact that the coalescence behavior is similar in these very different scenarios indicates the robustness of the phenomenon and suggests that the local dynamics near the point of coalescence are more important than the nature of the global flow field.
For smaller droplets corresponding to aspect ratio less than $\varepsilon<$ 0.5 , the critical capillary number for coalescence increases as the aspect ratio decreases. Droplets with small aspect ratio are not as strongly confined by the channel walls. In this case, the channel width is no longer an adequate estimate of the droplet curvature. Instead, the nonuniform velocity profile deforms the droplet, leading to a 'bullet' shape with higher curvature at the nose and a blunted shape at the rear. ${ }^{51}$ Therefore, the local radius of curvature is smaller at the point of coalescence for smaller droplets. For isolated droplet pairs in a four-roll mill device, $\mathrm{Hu}$ et al. show that the critical capillary number decreases with increasing droplet radius according to equation (1). ${ }^{19} \mathrm{We}$ compare our observations with this scaling argument by measuring the local curvature of the approaching droplets at the point of coalescence. Substituting the local radius of curvature for $a$ in equation (1) yields an estimate of the critical capillary number as a function of aspect ratio, represented by the dashed line in Fig. 4. The figure shows that this estimate agrees reasonably well with the observed critical capillary number, although the transition is slightly under predicted at the smallest values of $\varepsilon$. These observations taken together suggest that the confinement of droplets within the microchannels influences the coalescence process by determining the local speed and curvature of the approaching droplets. Once the local conditions are determined, the coalescence process proceeds as expected for idealized, unconfined droplets.

Finally, we examine the influence of viscosity ratio on coalescence in microchannels. Fig. 5 shows the observed critical capillary number as a function of viscosity ratio for droplets colliding in a microfluidic T-junction. For $\lambda<2, C a_{\mathrm{c}}$ exhibits power law dependence on viscosity ratio, with a fitted power law exponent of $-0.49 \pm 0.04$. Larger than $\lambda>2$, the critical capillary number increases dramatically. These observations are consistent with coalescence in other geometries. For isolated droplet

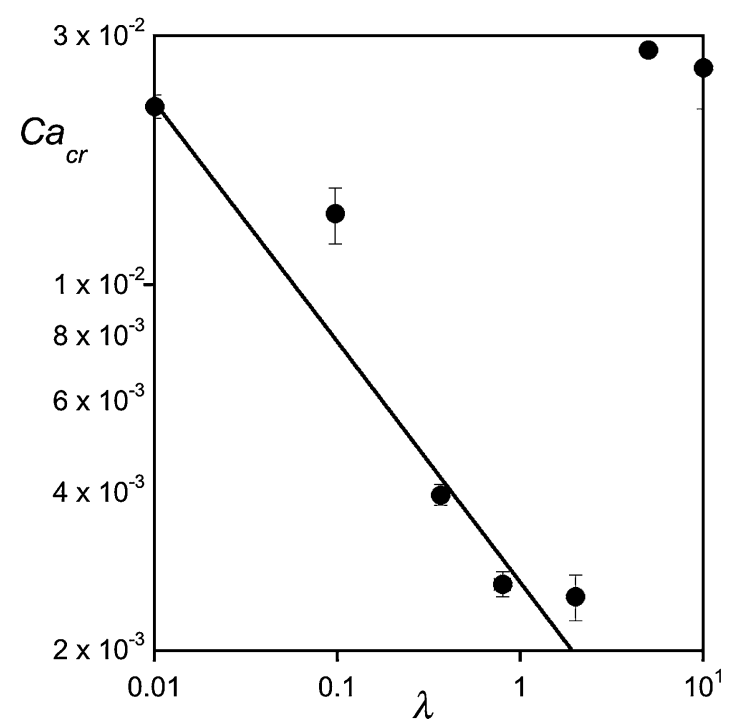

Fig. 5 Critical capillary number for coalescence $C a_{\text {crit }}$ as a function of viscosity ratio $\lambda$. The critical capillary number decreases with increasing viscosity ratio up to a viscosity ratio of $\lambda=1.3$, exhibiting power law behavior with an exponent of $-0.49 \pm 0.04$. The critical capillary number increases dramatically for the two largest viscosity ratios considered. 
pairs, Hu et al. show that the critical capillary number decreases with increasing viscosity ratio according to equation (1). ${ }^{19}$ Comparing our experiments with those of $\mathrm{Hu}$ et al. we find that the power law exponents agree with one another within the uncertainty of the two measurements. For droplet pairs colliding in constricted capillaries, Yan et al. also observe that the power law dependence given in equation (1) is maintained up to a limiting viscosity ratio of $\lambda<5 .{ }^{28}$ For larger viscosity ratios, the critical capillary number increases dramatically, consistent with our observations.

Yan et al. attribute the increase in the critical capillary number at larger viscosity ratios to the fact that viscous droplets are more resistant to deformation. Less viscous droplets are able to deform rapidly in a collision and create a larger interfacial area over which film drainage must occur, effectively slowing film drainage and inhibiting coalescence. Viscous droplets resist deformation, permitting film drainage to occur more rapidly over a smaller interfacial area. The authors also observe that viscous droplets tend to coalesce downstream of the constriction, while less viscous droplets coalesce within the constriction. ${ }^{28}$ Consistent with these simulations, we also observe that at the highest viscosity ratios, or lowest droplet viscosities, coalescence occurs at the nose of the droplets, where collision occurs. As the internal viscosity increases, the coalescence point moves further upstream of the nose.

\section{Critical capillary number for splitting}

The tendency for a droplet to split into two parts depends on its initial aspect ratio and the strength of the deformation induced by the collision. The Rayleigh Plateau criterion states that when the length of a droplet exceeds its circumference, or $\varepsilon=l / \pi w>1$, the droplet is unstable and any perturbation leads to droplet breakup. ${ }^{52}$ Thus, we expect that any droplet larger than $\varepsilon>1$ will break, as long as the capillary number exceeds the critical value for coalescence. Fig. 4 confirms this expectation, and shows that in fact, smaller droplets also break if the collision speed is fast enough. As the capillary number increases, the critical aspect ratio for breakup decreases, implying that more kinetic energy of the hitting droplet is needed to break a smaller receiving droplet.

This critical behavior resembles the response of a single droplet to the extensional flow field in a microfluidic T-junction. ${ }^{6}$ In this case, the droplet stretches and sometimes splits in the junction. Link et al. develop a stability argument based on the idea that the droplet shape exhibits power law dependence on the shear rate $G, l_{\mathrm{c}}-l \propto\left(G_{\mathrm{c}}-G\right)^{1 / 2}$ as it approaches the critical condition beyond which the elongated droplet shape is no longer steady..$^{53}$ These arguments lead to a stability criterion for splitting given by

$$
C a_{\mathrm{c}, \mathrm{split}}=\alpha \varepsilon\left(\varepsilon^{-2 / 3}-1\right)^{2}
$$

where $\alpha$ is a fitting parameter that depends on the viscosity ratio and the geometry of the splitting junction. The stability boundary given by equation (3) with $\alpha=0.75$ is indicated in Fig. 4 as a solid line. Although the flow conditions leading to splitting of a droplet are not identical in the present study to those of Link et al., the predicted stability boundary agrees reasonably well with the observed transition from slipping to splitting. These results indicate that when the hitting droplet squeezes the receiving droplet at the junction, the subsequent elongation of the receiving droplet is similar to that experienced by a single droplet in an elongational flow.

\section{Characterization of secondary behaviors}

The secondary responses to a collision, $c f$. Fig. 2(D)-(F), are observed less consistently than the primary responses, $c f$. Fig. 2(A)-(C). In the case of multiple splitting, the capillary number of the original splitting event is always well above the critical capillary number for coalescence. We assume that the conditions for subsequent splitting events must obey similar criteria to those described earlier. Late splitting tends to occur more frequently at higher viscosity ratios due to increased resistance to deformation. For example, Fig. 2(F) corresponds to $\lambda=5$. Late coalescence occurs at capillary numbers near the transition from coalescence to slipping and is only observed for small droplets with aspect ratio less than $\varepsilon<0.75$, as shown in the inset to Fig. 4. Late coalescence is analogous to observations in isolated droplets that coalescence can occur at angles greater than $45^{\circ}$, counter to expectations from a simple force balance. Although the droplets are beginning to separate in this case, Borrell et al. argue that a suction force is created in the thin draining film. ${ }^{25}$

\section{Conclusions}

Using a simple T-junction, we have demonstrated experimentally that droplet coalescence can occur easily in microfluidic devices without the need for external fields. The primary criterion for coalescence is that the collision speed must be low enough. At faster collision speeds, droplets can interact but not merge, or one droplet can split the other into two segments. Slipping is more likely for smaller droplets, while splitting occurs in larger droplets. We have found that the critical capillary number for coalescence depends on the local curvature of the colliding droplets at the point of collision, which depends on the speed of collision. The magnitude of the critical value and its dependence on the local curvature and the viscosity ratio agree well with that observed for both isolated and confined droplets.

Slipping and splitting occur at larger speeds at which coalescence is not favored. The transition between the two occurs at a critical capillary number that is a function of the initial droplet length. A stability criterion can be formulated in terms of the Rayleigh-Plateau criterion and the stability of droplets elongating in an extensional flow. The same criterion has been used previously in the context of a single droplet colliding a microfluidic T-junction. We note that the $90^{\circ}$ angle of the collision junction and the relatively narrow downstream channel promote splitting, a response that is not observed in the numerical simulations of Yan et al. for a contraction-expansion in a straight channel. The stability criterion probably depends on the collision angle and the downstream channel geometry, allowing for further control over this behavior in the design of droplet-based devices.

In summary, the conditions needed to obtain flow-induced coalescence at microfluidic junctions are completely consistent with prior observations for coalescence in other flow 
configurations, suggesting that the local speed and the local curvature of the droplets are the relevant controlling parameters. Observations at larger viscosity ratios and near the transitions between behaviors indicate that the time of contact between the droplets as they interact compared with the time required for film drainage determines the location along the interacting surface where coalescence or splitting occur.

While we have restricted our study to droplet pairs with nominally equal size and speed, we expect the same general observations to hold even when the two droplets are not identical. When the two droplets are not identical, the results will be more sensitive to which droplet arrives at the junction first. The operating diagram and transition criteria presented in this paper should enable the informed design of droplet-based lab-on-achip devices. A significant advantage of microfluidic devices is the possibility to fabricate complex geometries. For example, if high production frequencies are desired for high throughput applications, coalescence can still be achieved by reducing collision speeds via a larger local cross-sectional area near the collision. Tailoring the angle and downstream geometry of the collision junction can also help tune conditions to promote coalescence while avoiding splitting.

\section{Acknowledgements}

This research was partially supported by the Donors of the American Chemical Society Petroleum Research Fund and the Pennsylvania Infrastructure Technology Alliance. GFC thanks Philip L. and Marsha Dowd for partial support of his doctoral research through a Dowd-ICES Graduate Fellowship. In addition, we thank the Colloids, Polymers and Surfaces Laboratory and the CMU MEMS Laboratory for use of these facilities for device fabrication and fluid characterization. We are grateful to Lynn Walker and Steve Garoff for insightful comments that have led to significant improvements to this work.

\section{References}

1 H. Song, D. L. Chen and R. F. Ismagilov, Angew. Chem., Int. Ed., 2006, 45, 7336 .

2 M. A. Burns, B. N. Johnson and S. N. Brahmasandra, et al., Science, 1998, 282, 484

3 B. Zheng, L. S. Roach and R. F. Ismagilov, J. Am. Chem. Soc., 2003, 125,11170 .

4 V. Cristini and Y. C. Tan, Lab Chip, 2004, 4, 257.

5 G. F. Christopher and S. L. Anna, J. Phys. D: Appl. Phys., 2007, 40, R319.

6 D. R. Link, S. L. Anna and D. A. Weitz, et al., Phys. Rev. Lett., 2004, 92.

7 S. Y. Teh, R. Lin and L. H. Hung, et al., Lab Chip, 2008, 8, 198.

8 D. R. Link, E. Grasland-Mongrain and A. Duri, et al., Angew. Chem., Int. Ed., 2006, 45, 2556.

9 M. Chabert, K. D. Dorfman and J. L. Viovy, Electrophoresis, 2005, 26, 3706.

10 I. Shestopalov, J. D. Tice and R. F. Ismagilov, Lab Chip, 2004, 4, 316.
11 L. H. Hung, K. M. Choi and W. Y. Tseng, et al., Lab Chip, 2006, 6, 174.

12 Y. C. Tan, Y. L. Ho and A. P. Lee, Microfluid. Nanofluid., 2007, 3, 495.

13 K. Ahn, J. Agresti and H. Chong, et al., Appl. Phys. Lett., 2006, 88.

14 C. Priest, S. Herminghaus and R. Seemann, Appl. Phys. Lett., 2006, 89.

15 A. A. Garcia, A. Egatz-Gomez and S. A. Lindsay, et al., J. Magn. Magn. Mater., 2007, 311, 238.

16 L. G. Leal, Phys. Fluids, 2004, 16, 1833.

17 A. K. Chesters, Chem. Eng. Res. Des., 1991, 69, 259.

18 H. A. Stone, A. D. Stroock and A. Ajdari, Annu. Rev. Fluid Mech., 2004, 36, 381.

19 Y. T. Hu, D. J. Pine and L. G. Leal, Phys. Fluids, 2000, 12, 484.

20 F. Baldessari, G. M. Homsy and L. G. Leal, J. Colloid Interface Sci., 2007, 307, 188.

21 H. Yang, C. C. Park and Y. T. Hu, et al., Phys. Fluids, 2001, 13, 1087.

22 Y. Yoon, M. Borrell and C. C. Park, et al., J. Fluid Mech., 2005, 525, 355.

23 F. Baldessari and L. G. Leal, Phys. Fluids, 2006, 18.

24 M. A. Rother and R. H. Davis, Phys. Fluids, 2001, 13, 1178.

25 M. Borrell, Y. Yoon and L. G. Leal, Phys. Fluids, 2004, 16, 3945.

26 M. Loewenberg and E. J. Hinch, J. Fluid Mech., 1997, 338, 299.

27 M. R. Nobari, Y. J. Jan and G. Tryggvason, Phys. Fluids, 1996, 8, 29.

28 L. Yan, K. E. Thompson and K. T. Valsaraj, J. Colloid Interface Sci., $2006,298,832$.

29 R. S. Allan and S. G. Mason, J. Colloid Sci., 1962, 17, 383.

30 B. E. Burkhart, P. V. Gopalkrishnan and S. D. Hudson, et al., Phys. Rev. Lett., 2001, 87, 098304.

31 S. Guido and M. Simeone, J. Fluid Mech., 1998, 357, 1.

32 M. Loewenberg and E. J. Hinch, J. Fluid Mech., 1996, 321, 395.

33 A. Z. Zinchenko and R. H. Davis, Philos. Trans. R. Soc. London, Ser. A: Math. Phys. Eng. Sci., 2003, 361, 813.

34 X. Li and C. Pozrikidis, Phys. Fluids, 2002, 14, 61.

35 H. Aryafar and H. P. Kavehpour, Phys. Fluids, 2006, 18, 072105.

36 Z. Mohamed-Kassim and E. K. Longmire, Phys. Fluids, 2004, 16, 2170.

37 P. J. A. Janssen, P. D. Anderson and G. W. M. Peters, et al., J. Fluid Mech., 2006, 567, 65.

38 L. Y. Yeo, O. K. Matar and E. S. P. de Ortiz, et al., J. Colloid Interface Sci., 2003, 257, 93.

39 E. Klaseboer, J. P. Chevaillier and C. Gourdon, et al., J. Colloid Interface Sci., 2000, 274.

40 S. C. Case and S. R. Nagel, Phys. Rev. Lett., 2008, 1.

41 R. Manica, J. N. Connor and R. R. Dagastine, et al., Phys. Fluids, $2008,20$.

42 D. C. Duffy, J. C. McDonald and O. J. A. Schueller, et al., Anal. Chem., 1998, 70, 4974.

43 G. M. Whitesides and A. D. Stroock, Phys. Today, 2001, 54, 42.

44 P. Garstecki, M. J. Fuerstman and H. A. Stone, et al., Lab Chip, 2006, 6, 437.

45 J. Husny and J. J. Cooper-White, J. Non-Newtonian Fluid Mech., 2006, 137, 121.

46 G. F. Christopher, N. Noharuddin and S. L. Anna, Phys. Rev. E, 2008, Submitted.

47 V. Barbier, M. Tatoulian and H. Li, et al., Langmuir, 2006, 22, 5230.

48 G. Cristobal, J. P. Benoit and M. Joanicot, et al., Appl. Phys. Lett., 2006, 89.

49 W. L. Olbricht, Annu. Rev. Fluid Mech., 1996, 28, 187.

50 M. De Menech, Phys. Rev. E, 2006, 73.

51 A. Nadim and H. A. Stone, Stud. Appl. Math., 1991, 85, 53.

52 L. Rayleigh, Proc. R. Soc. London, 1879, 29, 71

53 Y. Navot, Phys. Fluids, 1999, 11, 990. 